\title{
ARTICLES
}

\section{Aggregation Issues in Cognitive Mapping}

\author{
Robert M. Kitchin \\ A. Stewart Fotheringham \\ Queen's University of Belfast \\ University of Newcastle
}

Cognitive mapping involves the description of the way individuals store and process geographic information. Typically, cognitive mapping data are derived from individual responses and then analyzed in one of three ways: (1) the individual data sets are analyzed separately and only pooled for comparison (disaggregation); (2) the individual data sets are averaged and then analyzed (collective aggregation); or (3) the individual data sets are analyzed and the results averaged (individual aggregation). This paper compares the latter two aggregation strategies for analyzing cognitive mapping data using data collected in a large-scale study of students' configurational knowledge of the city of Swansea in the United Kingdom. It is contended that the aggregation strategy adopted will have a fundamental effect upon the conclusions drawn from a study. Key Words: aggregation, cognitive mapping, ecological fallacy.

\section{Introduction}

$\mathrm{W}$ e have implicitly directed attention to the aggregation problem. On what basis does the behavioral geographer validly aggregate? It is not too strong to say we know virtually nothing about how aggregation ... affects the results of a study. And how can we examine this question until we can identify behavioral characteristics and empirically and theoretically test the consequences of different aggregation schemes? Surely this is the most pressing problem of behavioral researchers. (Golledge and Stimson 1987, 313)

The need to know about the world around us is fundamental to our daily lives. Each of us gathers and processes information to be used in decisions and choices regarding our spatial behavior. To make sense of the information gathered, it is hypothesized that we organize in our minds an understanding of the world in which we live (Kitchin 1994a). Cognitive mapping concerns the study of how we consciously, and more commonly subconsciously, acquire, learn, develop, think about, and store data relating to our everyday geographic environment (Downs and Stea 1973a). In recent years, a wide diversity of techniques has been used to gain an understanding of the way an individual comprehends part of the world. These techniques can be divided into those that gather data relating to distance and direction and those that produce some sort of a cartographic representation (Kitchin and Jacobson, forthcoming). Examples of the latter could include asking individuals to locate certain places on a map, to draw a route between two places, or to draw a map of a city showing the major landmarks. Whatever the task, the result is a set of individual cognitive data that needs to be summarized in some way in order to make some general statements about spatial cognition. There are three primary ways of producing such generalizations, as shown in Figure 1.

The first of these is disaggregation, where the data for each individual are analyzed separately and the results are pooled only for comparison (Fig. 1a). As a result, individual cases are still identified and differences between individuals can be noted. The second strategy, collective aggregation, requires the data to be aggregated prior to analysis so that only a single analysis takes place on the aggregated data (Fig. 1b). The results from the pooled data are taken to represent the whole group and there is no possibility of observing individual-level results. In the third strategy, that of individual aggregation, the data are analyzed at the individual level but the results are both pooled and averaged and either the mean or median values are taken to represent the members of the whole group (Fig. 1c).

Because the aggregation strategies are different, it is likely that the results from them will differ, leading to weak internal validity (the possibility of drawing alternative conclusions from the same data). As such, depending on the aggregation strategy used, fundamentally different conclusions could be drawn from the same data set, leading the researcher(s) to either under- or

Professional Geographer, 49(3) 1997, pages 269-280 () Copyright 1997 by Association of American Geographers. 
270 Volume 49, Number 3, August 1997

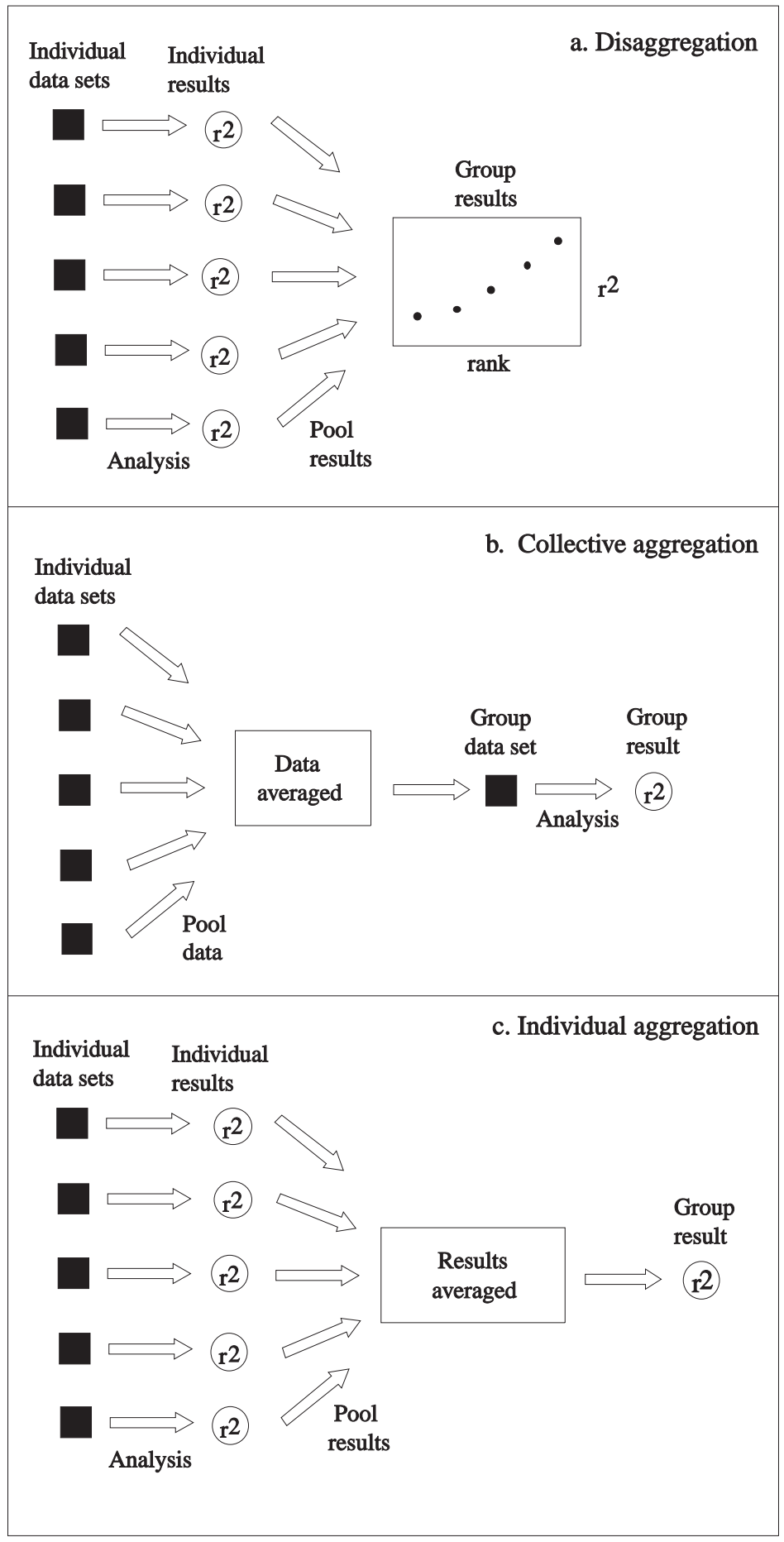

Figure 1: Strategies of analysis. 
overestimate the cognitive map knowledge of a group of individuals. The effect has been noted by Ewing (1981), but there has been no largescale examination of this issue. This paper compares the results of aggregating individual cognitive mapping data using both aggregation strategies described above, critically appraising their usefulness in analyzing cognitive mapping data sets.

\section{Aggregation Strategies for Cognitive Mapping Data}

While it has long been recognized that the results of any spatial analysis using aggregatelevel data will be sensitive to the definition of the areal units for which the data are reported (Gehlke and Biehl 1934; Neprash 1934), there has been a recent resurgence of interest in this topic (Openshaw 1983; Fotheringham and Wong 1991; and Fotheringham et al. 1995). These studies examine the sensitivity of analytical results to modifications in the way areal units are defined and typically show that different conclusions can be drawn from the same data when they are aggregated either to different levels (a scale effect) or in different zones at the same scale (the zoning effect). This paper has a different emphasis in that it examines the sensitivity of cognitive mapping results to the method of aggregation. In this paper we examine two different ways in which individual responses might be aggregated, defined in Figures $1 \mathrm{~b}$ and 1c, in order to identify generalities regarding spatial cognition.

There is evidence to suggest that the issue of aggregation is especially important in cognitive studies. Bryant (1984, 43), for example, concluded that there is "little evidence . . . to suggest . . . a unified mental representation of the geographical environment," suggesting that although individuals may experience the same social and physical characteristics, large differences in cognitive map knowledge still exist between respondents. Aggregation, therefore, is likely to mask the true cognitive map knowledge of individuals. Thus, Lloyd (1989, 123) suggests that "averaging coordinate data for individual subjects to determine the characteristics of typical individual cognitive maps may not accurately represent either the severity or character of distortions in such maps." Harman and Betak
(1976) argued that this is because the evidence that groups share the same socioeconomic, ethnic, or familiarity characteristics is circumstantial and does not mean that groups share the same cognitive structures. They further argued (Harman and Betak 1976, 14) that "there is a real need to reexamine ... the extent to which it is meaningful to aggregate across individual differences."

Alternatively, Gould (1975) argued that individual responses are not particularly meaningful except at a highly personal level, and it is only through aggregation that distinct, interpretable patterns emerge. He supplemented this (Gould 1976) by arguing that an understanding of the individual's spatial behavior does not give a comprehensive understanding of how a group of individuals act as a system, when we so obviously share the same spatial behavior patterns. Downs and Stea (1973b) contended that individuals within a group can be aggregated because the spatial environment experienced contains many regular and recurrent features with spatial behavior patterns of individuals displaying similar origins, destinations, and frequency of movement. Additionally, they argued that people share common information, processing capabilities, and strategies. These capabilities are associated with innate, physiological parameters of human information processing, while the common strategies are learned methods of coping with the environment. Thus people experience the environment in similar ways, using common experiential models of geographic space.

To date, most cognitive mapping data have been analyzed using the collective aggregation strategy (Magana et al. 1981; Golledge et al. 1985; Lloyd 1989). Little use has been made of the individual aggregation method, although this is not surprising given that both analytical behavioral geography and environmental psychology are "positivistic" in nature. Certainly, until recent developments in computing power it would have been unrealistic to analyze the data at an individual scale due to logistical constraints. However, it is now possible to quickly and efficiently analyze large numbers of individual data sets. It is therefore the intention of this study to determine whether there are likely to be sufficient differences in the conclusions 
drawn from these two aggregation strategies and to comment critically on their respective advantages and disadvantages.

\section{The Experimental Data}

The data used were collected as part of a larger study assessing 170 students' configurational knowledge of the Swansea region in the United Kingdom. In this larger study, students were divided into 6 groups who each completed 4 tests from a total of 13 tests. In all, 52 students were asked to locate 25 places in the region using two different spatial cued response (SCR) tests (Fig. 2). In the first (SCR1), 19 students were given only the locations of the Geography Department at the University of Wales Swansea and the bus station to guide their placements. In the second (SCR2), 33 students were also given the coastline to guide their responses. Given that respondents were matched for educational ability and familiarity (all had been resident in Swansea for 9-10 weeks), one might expect the responses to be more accurate in the second test where more spatial information was provided.
To fully explore any potential aggregation effects caused by the aggregation strategy used, the two sets of experimental data (SCR1 and SCR2) were analyzed at four different levels. The resulting sub-data sets are differentiated in terms of the number of locations they contain (Table 1). The data sets are progressively ordered with data of increasing familiarity removed at each level. Data set A contains data on the cognitive locations of all 25 places. In data set $\mathrm{B}$, the locations of all places reported as "guessed" by the respondents were eliminated. In data set $\mathrm{C}$, those places that scored a familiar rating less than 2 (unknown or low familiarity places) were excluded; and in data set $\mathrm{D}$, those places scoring a familiar rating less than 4 (unknown, low, and medium familiarity places) were excluded to leave those places which were very familiar. All the data sets were analyzed using bidimensional regression, which is essentially ordinary least squares regression extended to two dimensions (Tobler 1965), with cognized locations bidimensionally regressed into real world locations. This was performed using the CMAP package (Kitchin 1994b).

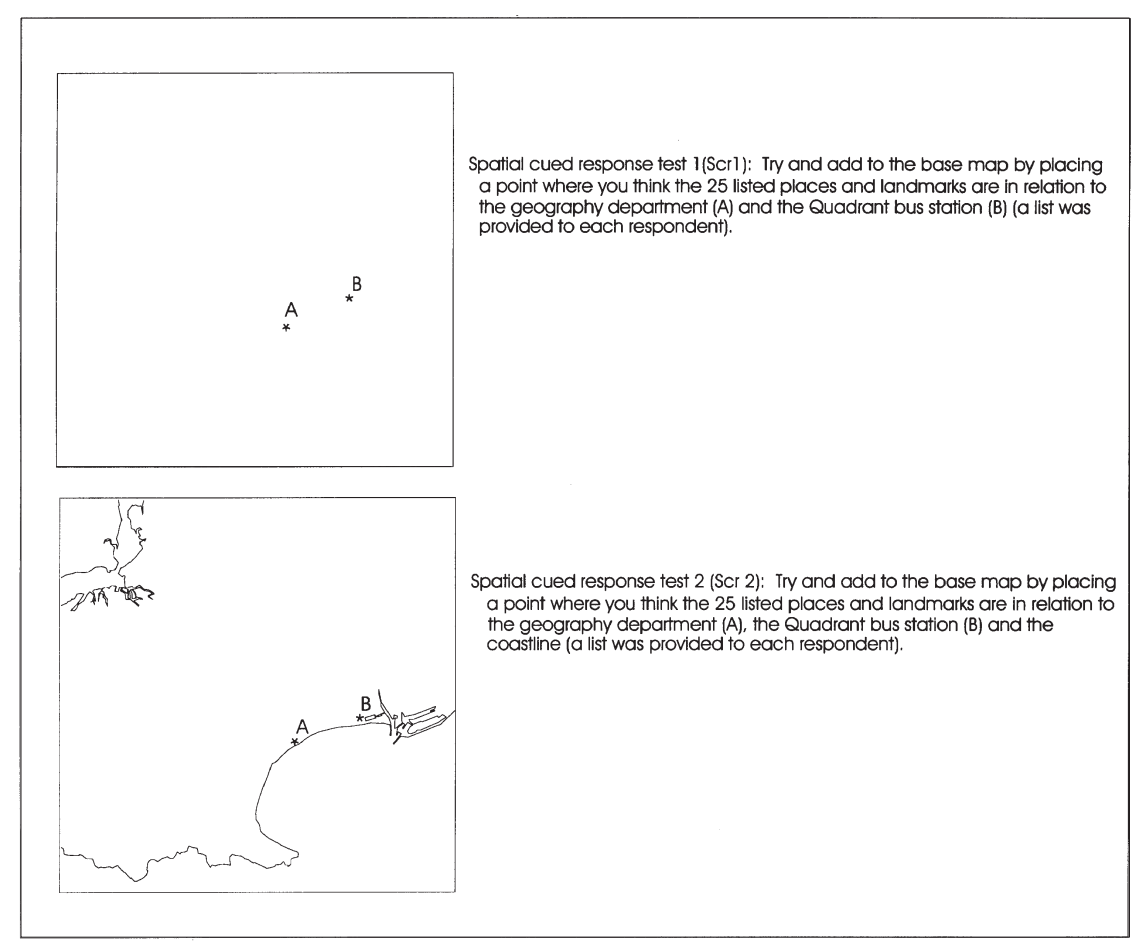

Figure 2: The tests used to investigate aggregation. 
Table 1 Experimental Data Sets

\begin{tabular}{lcc}
\hline & $\begin{array}{c}\text { Data set SCR 1 (No Coastline) } \\
\text { (No Coastline) }\end{array}$ & $\begin{array}{c}\text { Data set SCR 2 } \\
\text { (Coastline) }\end{array}$ \\
\hline Sub-data set A & 25 places located & 25 places located \\
Sub-data set B & Guesses removed & Guesses removed \\
Sub-data set C & Low familiar places removed & Low familiar places removed \\
Sub-data set D & Low and medium places removed & Low and medium places removed \\
\hline
\end{tabular}

\section{Results}

Tables 2 and 3 provide the aggregated values for all the bidimensional regression results for each of the four data sets (A-D) for the two spatial cued response tests. The result variables in the tables represent different facets of the goodnessof-fit between the real world and cognitive data sets. The $\mathbf{r}^{2}$ value represents the measure of association between the two configurations. The scale is an index that measures the scale change needed to produce the best fit between the cognitive space and the real world space. A scale value of less than one indicates that the cognitive space needs to be contracted (reduced) to fit the real world space; a scale value of greater than one indicates that the cognitive space needs to be expanded. The angle value is the angle to which the coordinate axes must be rotated to produce the best fit with the real world space. An angle value with a positive value indicates the counterclockwise rotation needed to produce a best fit; a negative value indicates the clockwise rotation. The $\mathbf{a}_{\mathbf{1}}$ value is the horizontal translation, with a positive value indicating a west-toeast shift and a negative value indicating an east-to-west shift; $\mathbf{a}_{2}$ is the vertical translation, with a positive value indicating a south-to-north shift and a negative value indicating a north-tosouth shift (Lloyd 1989). The distortion index

Table 2 Spatial Cued Response Test 1 Aggregated Results for Four Data Sets

\begin{tabular}{|c|c|c|c|c|c|c|c|c|}
\hline \multirow[t]{3}{*}{ Variable } & \multicolumn{4}{|c|}{ Data set A } & \multicolumn{4}{|c|}{ Data set B } \\
\hline & \multirow{2}{*}{$\frac{\mathrm{CA}}{\text { mean }}$} & \multicolumn{3}{|c|}{ IA } & \multirow{2}{*}{$\frac{\mathrm{CA}}{\text { mean }}$} & \multicolumn{3}{|c|}{$\mathrm{IA}$} \\
\hline & & mean & med & $\overline{\text { st d }}$ & & mean & med & st d \\
\hline$r^{2}$ & .77 & .55 & .59 & .18 & .82 & .67 & .72 & .13 \\
\hline scale & 0.96 & 0.81 & 0.83 & 0.16 & 0.95 & 0.82 & 0.82 & 0.16 \\
\hline angle & -24.21 & -25.65 & -26.42 & 8.93 & -22.17 & -23.56 & -24.79 & 8.92 \\
\hline$a_{1}$ & 285.56 & 351.2 & 363.7 & 107.9 & 272.39 & 327.8 & 309.8 & 106.9 \\
\hline$a_{2}$ & -194.99 & -99.5 & -118.4 & 130.1 & -174.26 & -104.1 & -75.7 & 132.5 \\
\hline distortion index & 35.30 & 50.42 & 48.20 & 12.29 & 30.51 & 42.20 & 38.88 & 9.89 \\
\hline box side 1 & 225.27 & 375.5 & 373.8 & 122.5 & 232.54 & 299.5 & 270.8 & 96.6 \\
\hline box side 2 & 397.24 & 508.3 & 513.8 & 159.1 & 318.94 & 332.3 & 330.1 & 147.3 \\
\hline absolute distortion & 2382 & 2929 & 2878 & 1003 & 2314 & 2317 & 2200 & 1065 \\
\hline relative distortion & 1721 & 2436 & 2544 & 685 & 1527 & 1646 & 1747 & 625 \\
\hline total distortion & 2676 & 3643 & 3481 & 1072 & 2535 & 2704 & 2741 & 1169 \\
\hline \multirow[t]{3}{*}{ Variable } & \multicolumn{4}{|c|}{ Data set C } & \multicolumn{4}{|c|}{ Data set D } \\
\hline & \multirow{2}{*}{$\frac{\mathrm{CA}}{\text { mean }}$} & \multicolumn{3}{|c|}{$\mathrm{IA}$} & \multirow{2}{*}{$\frac{\mathrm{CA}}{\text { mean }}$} & \multicolumn{3}{|c|}{$\mathrm{IA}$} \\
\hline & & mean & med & $\overline{\text { st d }}$ & & mean & med & st d \\
\hline$r^{2}$ & .85 & .69 & .72 & .16 & .80 & 72 & .72 & .16 \\
\hline scale & 0.94 & 0.82 & 0.80 & 0.14 & 0.88 & 0.84 & 0.82 & 0.18 \\
\hline angle & -22.86 & -22.00 & -17.39 & 10.79 & -19.98 & -17.64 & -19.30 & 14.74 \\
\hline$a_{1}$ & 276.20 & 317.8 & 316.8 & 111.3 & 270.59 & 262.2 & 280.9 & 160.9 \\
\hline $\begin{array}{l}a_{1} \\
a_{2}\end{array}$ & -189.65 & -99.3 & -60.4 & 135.3 & -136.35 & -75.7 & -60 & 161.2 \\
\hline distortion index & 28.22 & 39.79 & 38.98 & 12.30 & 32.06 & 37.53 & 38.89 & 12.41 \\
\hline box side 1 & 233.08 & 232.5 & 221.2 & 99.4 & 275.73 & 175.1 & 145.5 & 85.7 \\
\hline box side 2 & 281.07 & 226.4 & 191.0 & 128.5 & 250.45 & 150.4 & 106.6 & 89.6 \\
\hline absolute distortion & 2393 & 1569 & 1385 & 951 & 2067 & 968 & 1102 & 528 \\
\hline relative distortion & 1513 & 1099 & 975 & 593 & 1464 & 687 & 797 & 352 \\
\hline total distortion & 2546 & 1780 & 1482 & 1010 & 2318 & 1062 & 1171 & 519 \\
\hline
\end{tabular}

CA - data aggregated

IA - individual aggregated

med-median

st $d$ - standard deviation 
274 Volume 49, Number 3, August 1997

Table 3 Spatial Cued Response Test 2 Aggregated Results for Four Data Sets

\begin{tabular}{|c|c|c|c|c|c|c|c|c|}
\hline \multirow[t]{3}{*}{ Variable } & \multicolumn{4}{|c|}{ Data set A } & \multicolumn{4}{|c|}{ Data set B } \\
\hline & \multirow{2}{*}{$\frac{\mathrm{CA}}{\text { mean }}$} & \multicolumn{3}{|c|}{ IA } & \multirow{2}{*}{$\frac{\mathrm{CA}}{\text { mean }}$} & \multicolumn{3}{|c|}{$\mathrm{IA}$} \\
\hline & & mean & med & st d & & mean & med & st d \\
\hline$r^{2}$ & .79 & .52 & .59 & .25 & .85 & .67 & .75 & .22 \\
\hline scale & 1.17 & 0.87 & 0.94 & 0.87 & 1.12 & 0.91 & 0.94 & 0.14 \\
\hline angle & -5.32 & -5.11 & -4.36 & -5.11 & -5.31 & -5.75 & -4.08 & 10.75 \\
\hline$a_{1}$ & -47.81 & 114.24 & 91.20 & 114.24 & -29.71 & 91.9 & 56.4 & 127.0 \\
\hline$a_{2}$ & -122.04 & 24.40 & 22.42 & 24.40 & -105.96 & -9.9 & -7.2 & 82.1 \\
\hline distortion index & 33.56 & 52.18 & 48.33 & 52.18 & 27.78 & 41.60 & 36.32 & 16.06 \\
\hline box side 1 & 169.44 & 408.46 & 403.50 & 408.46 & 128.37 & 296.6 & 251.3 & 128.7 \\
\hline box side 2 & 399.74 & 517.01 & 509.86 & 517.01 & 335.80 & 328.9 & 343.2 & 151.7 \\
\hline absolute distortion & 1007.43 & 1370.5 & 1321.2 & 1370 & 836.24 & 966 & 784 & 665 \\
\hline relative distortion & 1579.71 & 2384 & 264.4 & 2384 & 1340 & 1562 & 1456 & 578 \\
\hline total distortion & 1455.36 & 2431.8 & 2390.6 & 2431 & 1215 & 1652 & 1422 & 681 \\
\hline \multirow[t]{3}{*}{ Variable } & \multicolumn{4}{|c|}{ Data set C } & \multicolumn{4}{|c|}{ Data set D } \\
\hline & CA & \multicolumn{3}{|c|}{ IA } & \multirow{2}{*}{$\frac{\mathrm{CA}}{\text { mean }}$} & \multicolumn{3}{|c|}{$\mathrm{IA}$} \\
\hline & mean & mean & med & st d & & mean & med & st d \\
\hline$r^{2}$ & .89 & .78 & .84 & .19 & .93 & .79 & .86 & .20 \\
\hline scale & 1.06 & 0.93 & 0.96 & 0.11 & 1.00 & 0.93 & 0.92 & 0.16 \\
\hline angle & -4.31 & -2.34 & -1.13 & 7.07 & -5.00 & -2.49 & -0.45 & 7.36 \\
\hline$a_{1}$ & 4.81 & 65.6 & 51.4 & 83.5 & 43.55 & 69.3 & 72 & 100.3 \\
\hline$a_{2}$ & -73.68 & 4.5 & 3.6 & 86.3 & -62.01 & -4.5 & 10.3 & 109.2 \\
\hline distortion index & 23.29 & 32.59 & 28.57 & 14.89 & 18.71 & 30.45 & 27.44 & 15.97 \\
\hline box side 1 & 95.13 & 182.5 & 157.1 & 92.9 & 118.58 & 131.8 & 129.7 & 89.5 \\
\hline box side 2 & 285.98 & 200.3 & 159.3 & 138.9 & 221.15 & 122 & 104 & 73.8 \\
\hline absolute distortion & 576.98 & 583 & 453 & 585 & 513.78 & 468 & 341 & 568 \\
\hline relative distortion & 1150 & 845.6 & 806.2 & 459.9 & 920.34 & 523.4 & 494.5 & 379.3 \\
\hline total distortion & 1046 & 923.7 & 822.6 & 555 & 958.16 & 622 & 487 & 532 \\
\hline $\begin{array}{l}\text { CA - data aggregate } \\
\text { IA - individual aggres } \\
\text { med-median } \\
\text { st } d \text { - standard devia }\end{array}$ & & & & & & & & \\
\hline
\end{tabular}

provides a measure of how distorted the cognitive configuration is on a scale between 0 and 100 and is the ratio of $\mathrm{D}$ to $\mathrm{D}_{\max }$, where $\mathrm{D}$ is the average distance of vectors separating matched pairs and $D_{\max }$ is the maximum value $\mathrm{D}$ can achieve, which is constrained by the objective map size (Waterman and Gordon 1984). This can be displayed graphically using a box of distortion that indicates the orientation of maximum and minimum distortion for each point whether by stretching or compressing, and the box's diagonal is the distortion distance $\mathrm{D}$. The absolute distortion refers to the distance between the real and cognitive locations and the relative distortion indicates whether the locations in the cognitive configuration are positioned relative to each other. The total distortion combines both of these elements. Each of these bidimensional regression variables reveals a different facet about the association between the cognitive space and real world space and allows the exact reason for any differences between spaces to be determined. For example, $\mathbf{r}^{2}$ values for two different individual data sets might be very similar, but the two data sets might differ significantly in terms of the scaling (scale), rotation (angle), and translation ( $\mathrm{a}_{1}$ and $\mathrm{a}_{2}$ ) needed to produce the best fit. Similarly, while some variables such as scale and angle might be contiguous, patterns of distortion might differ substantially across cognitive space. It is therefore important to examine all of these variables in order to fully understand individual and collective cognitive spaces and their relationship to real world space.

The four major columns of data refer to the four data sets used in which differences between cognized and actual locations are analyzed for different numbers of places. For each data set, results are reported for both the collective aggregation scheme and the individual aggregation scheme. For the latter, where individual results are averaged, both the mean and median results for all respondents completing each respective test are reported. The standard deviation across the respondents is also reported. In 
all cases the most obvious comparison to make is between the CA (collective aggregation) score and the mean IA (individual aggregation) score, although the median and standard deviation figures for the IA method are also informative because they show the degree of deviation in individual estimates.

It is clear that the two aggregation strategies produce different results for all four sub-data sets on both spatial cued response tests (Fig. 3). For example, the collective aggregation $\mathrm{r}$ squared value for data set A for SCR1 (Table 2) is .77. The equivalent individual aggregate value is .55 with a standard deviation of .18 . A similar difference is observed in Table 2 for SCR2. Indeed, Figure 4 shows that in both tests the vast majority of the individual $r$-squared values are lower than the respective aggregate CA values. In both tests, but particularly in SCR2 where the coastline was provided, the aggregated values disguise a great deal of variation in the individual responses, with some individuals clearly having an accurate cognition of the environment and others having very poor mental representations of the area.

A comparison of the $\mathrm{r}$-squared values across the four sub-data sets (A-D) reveals that the difference between the two aggregation strategies tends to decrease as the more unfamiliar locations are excluded from each individual's responses (Fig. 3). For instance, in data set D for SCR1, the r-squared value for the CA framework is .80 compared with a value of .72 with a standard deviation of .16 for the IA framework. As expected, the r-squared values tend to increase as the more unfamiliar places are dropped from the analysis, and comparing equivalent values and the cognitive maps appears to be more accurate when more spatial cueing is provided (Fig. 3). However, this result is itself a product of aggregation, as the individual results shown in Figure 4 suggest. It would appear from the individual $r$-squared values for SCR2, where more spatial information is provided in the form of the local coastline, that this extra information has the effect of producing a much greater variation in the accuracy of the cognitive maps. However, the extra spatial information is not leading to more accurate aggregated results as might be expected. The reason for this is that respondents are being asked to locate 25 places regardless of whether they are familiar with each place. Once the guesses (data set B) and unfamil- iar places (data sets $C$ and $D$ ) have been removed from the analysis the effect of the spatial cueing becomes apparent, with large increases in individual r-squared values and associated rises in IA and CA aggregated r-squared values (Figs. 3 and 4; Tables 2 and 3).

There are similar differences between the CA and IA methods of analysis on all the reported statistics in Tables 1 and 2. In most cases the results suggest that the interpretations regarding cognitive process would differ according to which method was employed. For example, the CA scale values are higher than the IA scale values. The IA scale values indicate that on average, the cognitive space of individual maps needs to be enlarged to best fit the real world space. The process of collective aggregation has removed this scaling effect. The differences between CA and IA values are consistent across the bidimensional regression variables, with no one variable being affected more than the others. However, the differences between the CA and IA angle values are negible. This is because for both SCR1 and SCR2 the coastline acts as a strong frame of reference by which to orientate placements. For SCR2 the coastline was provided to the respondents. On SCR1, the coastline was the dominant, mental, frame of reference. Students generally believed that the coastline was "shallower" than in reality, and as a consequence, orientated all their placements to fit this notion. There is little deviation between CA and IA values because of the consistency of this miscognition, with the majority of respondents believing the bay to be orientated along an east-west orientation.

Generally, the CA method produces a more stable set of results as the more unfamiliar places are excluded from the data set, with the IA method showing more clearly the improvement in individuals' cognitive maps as more unfamiliar places are excluded from the analysis. For instance, the total distortion measure reported for both SCR1 in Table 1 and SCR2 in Table 2 exhibits very little change across the four data sets when it is computed by the CA method but shows a rather dramatic change when computed by the IA method (the value for data set D being less than a third that for data set A in both cases) (Fig. 3). Again, this suggests that the more usual method of analysis, the CA method, which involves aggregating the data prior to analysis, can disguise 

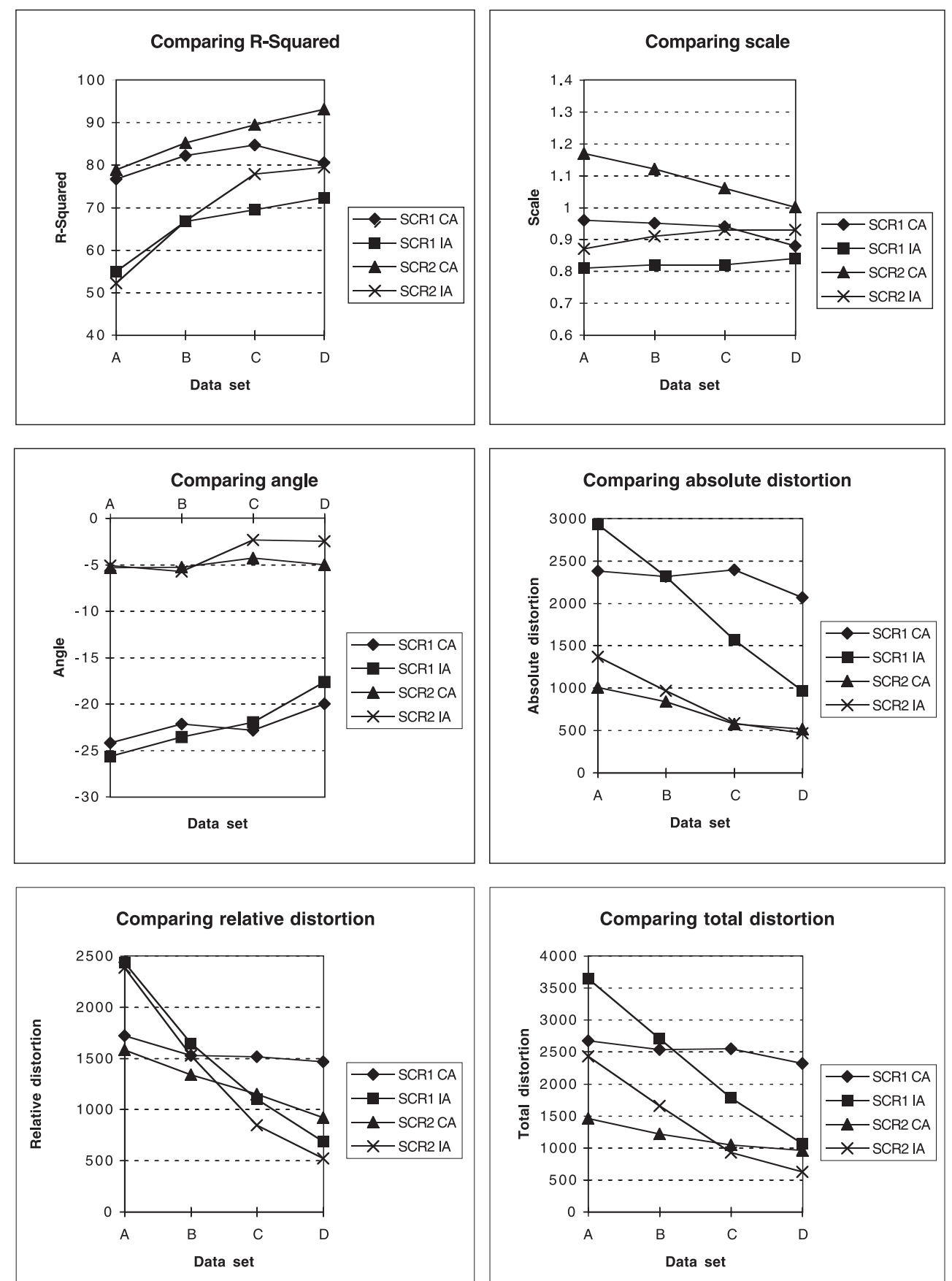

Figure 3: Comparing selected bidimensional regression results across sub-data sets. 
some interesting facets of cognitive mapping that are apparent only in the IA results by removing individual idiosyncratic differences.

However, although the above results suggest that the collective aggregation strategy needs to be used with caution when examining cognition, the strategy does allow groups of individuals to be compared on the basis of their shared place cognition. Place cognition is the consensual view of where each individual place is located. Through collective aggregation, a group's underlying, shared cognitive map knowledge can be investigated, with the unique individual deviations removed. It is argued that any remaining systematic distortions will be part of normal processing and part of a group's cognitive map
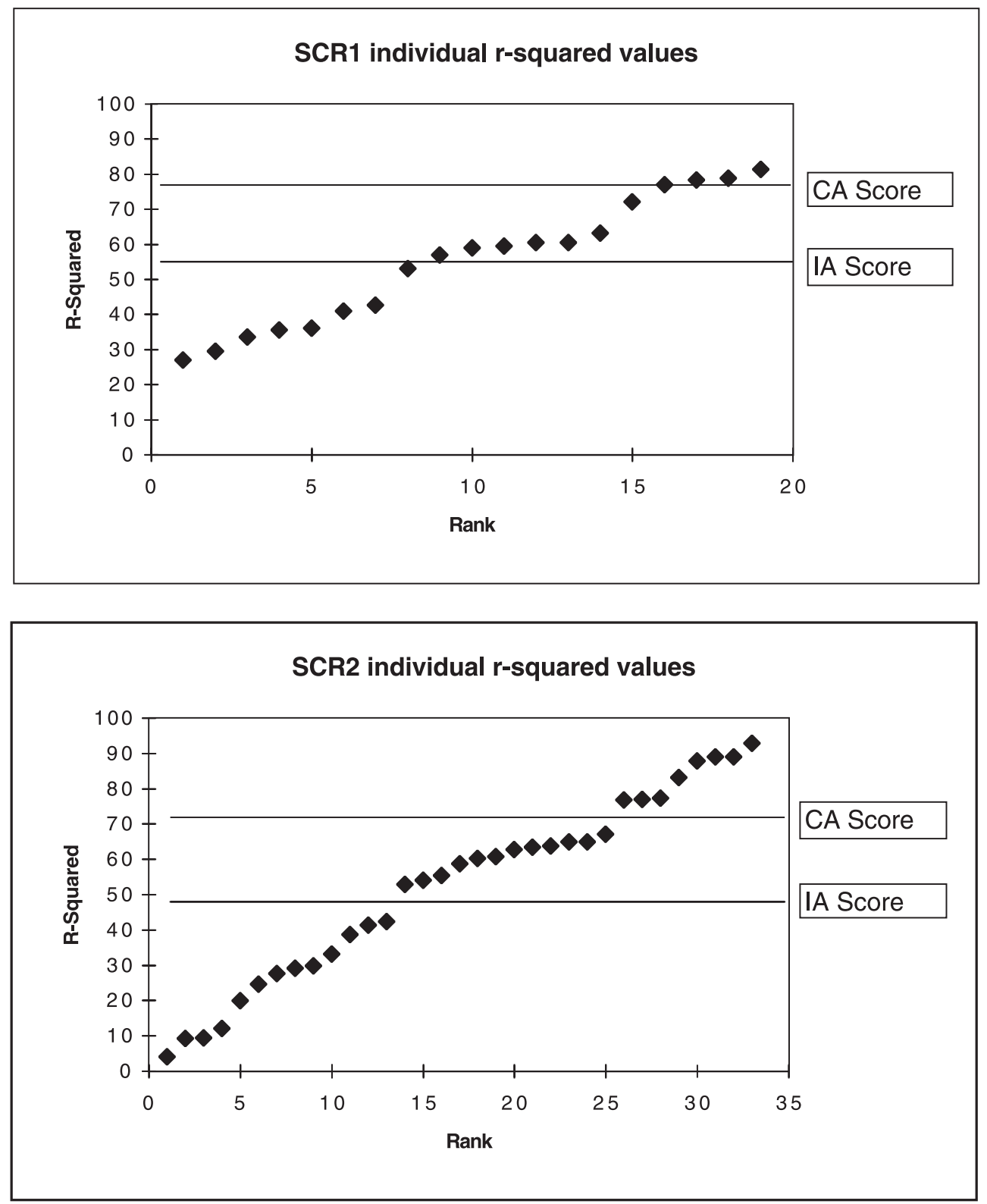

Figure 4: R-squared values from the spatial cured response tests. 
knowledge (Lloyd and Heivly 1987). Thus the method allows the group's cognition of the base data to be assessed, although there may well be problems of making inferences about the group's individual cognition. A similar finding in different contexts has been reported by, inter alia, Newcombe (1985), Cadwallader (1976), Mackay and Olshavsky (1975), and Lloyd (1989).

\section{Significance of Differences}

The fact that the two different aggregation strategies produced differing results and hence alternative conclusions when used to analyze the same data sets has important implications relating to validity and integrity of a study. In particular, analytical and ecological validity become weakened. Analytical validity concerns whether the most appropriate method of analysis has been chosen, leading to results that are conceptually or statistically sound and represent the data truly (Turk 1990). For example, McNamara et al. (1989) suggest that some methods of analysis might impose a structure on the results that in reality does not exist. The example they use to highlight this problem is that of an ordered tree algorithm used to determine if data are hierarchically structured. This approach, they argue, might identify a hierarchical structure in the data, where in fact only clustering across an area takes place. Thus in some cases this analysis leads to erroneous conclusions because the results cannot be interpreted with confidence.

Ecological validity is concerned with the inferences that can be made from the results of a study. In psychology, it generally refers to the extent to which environments experienced by the respondents in a scientific investigation have the properties they are supposed to have, or are assumed to have by the investigator (Brofenbrenner 1979). In geography, however, ecological validity generally concerns the problems of inferring characteristics of individuals from aggregate data referring to a population (Johnston et al. 1991). There are a number of other fallacies associated with ecological validity concerning the problems of generalization across circumstances, times, and areas (Alker 1969). Individualistic fallacy is the assumption that a whole is no more than the sum of its parts; many societies are more than mere aggregations of individuals and the characteristics of the latter should not necessarily be attributed to the former. Universal (external) fallacy assumes that the pattern observed in a sample holds for all, but very often, the data set studied is not a random sample of the population to which the results are generalized. Selective fallacy occurs when the experimenter uses carefully selected cases to "prove" a general point. Cross-sectional fallacy is the assumption that what is observed at one point in time holds for others. Crosslevel fallacy assumes that a pattern observed for one aggregation of the data will hold for all aggregation (using the same strategy) with differences in scale and zonation potentially affecting results (modifiable areal unit problem [MAUP]).

This study has amply demonstrated that there also exists an analytical fallacy, which assumes that the pattern observed for one aggregation of the data will hold when a different aggregation strategy is used. This is clearly not the case. As such, we have shown that, at present, both the analytical and ecological validity of cognitive mapping studies using an aggregation strategy are weakened. However, by identifying the strengths and weaknesses of each strategy it has been demonstrated that each strategy can be used with confidence to explore particular trends within the data. As a result, it is suggested that care needs to taken to choose the appropriate aggregation strategy when analyzing cognitive mapping data sets. Blindly adopting the traditional collective aggregation strategy could potentially lead to weakened validities and to a study with low integrity, from which few definitive conclusions can be drawn.

\section{Conclusions}

Clearly, care has to be exercised in drawing conclusions from aggregated cognitive mapping data. The strategy of collective aggregation is useful for measuring place cognition, but no inferences concerning the cognitive maps of individuals (individual cognition) can be drawn from such a strategy of analysis because it exaggerates individual cognition by removing residual errors. As such, cognitive mapping studies that have employed the strategy of collective aggregation to draw conclusions about individuals' cognitive maps might be misleading. For instance, Magana et al. (1981) used the strategy of collective aggregation individual coordinate data to compare four different tests designed to measure configurational knowledge. As noted, such a strategy is flawed because collective 
aggregation removes residual error producing $\mathbf{r}^{2}$ values that are similar. It is shown above from the disaggregated results that tests that have similar data aggregated results can produce different disaggregated results (Fig. 3). Evans et al. (1981) used the strategy of collective aggregation to compare groups of individuals at two different sites and over time, concluding that individuals did improve with time. Again, because this study has drawn conclusions about the individual cognition of a group's members from collective aggregated results, its conclusions may be misleading.

In summary, given that limitations in computing no longer apply and that cognitive mapping studies collect individual response data, the focus of the study should drive the type of aggregation strategy employed. If the focus of the study is on the overall cognition of a place, collective aggregation such as in Curtis and Fotheringham (1995) is possibly the preferred strategy. However, if the focus of the study is on a group of individuals' cognitive map knowledge, the preferred type of analysis is that employing individual aggregation.

\section{Literature Cited}

Alker, H. S. 1969. A typology of ecological fallacy. In Quantitative Ecological Analysis in the Social Sciences, ed. M. Dogan and S. Rokan, 69-86. London: MIT Press.

Brofenbrenner, U. 1979. The Ecology of Human Development. Cambridge, MA: Harvard University Press.

Bryant, K. J. 1984. Methodological convergence as an issue within environmental cognition research. Journal of Environmental Psychology 4: 43-60.

Cadwallader, M. T. 1976. Cognitive distance in intraurban space. In Environmental Knowing, ed. G. T. Moore and R. G. Golledge, 316-24. Stroudsburg, PA: Dowden, Hutchinson \& Ross.

Curtis, A., and A. S. Fotheringham. 1995. Large scale information surfaces: An analysis of city name recalls in the United States. Geoforum 26: 65-78.

Downs, R. M., and D. Stea. 1973a. Theory. In Image and Environment, ed. R. M. Downs and D. Stea, 8-26. Chicago: Aldine.

- 1973b. Cognitive maps and spatial behavior: Process and products. In Image and Environment, ed. R. M. Downs and D. Stea, 8-26. Chicago: Aldine.

Evans, G. W., D. G. Marrero, and P. A. Butler. 1981. Environmental learning and cognitive mapping. Environment and Behavior 13: 83-104.
Ewing, G. O. 1981. On the sensitivity of conclusions about the bases of cognitive distance. Professional Geographer 35: 311-14.

Fotheringham, A. S., and D. W. S. Wong. 1991. The modifiable areal unit problem in multivariate statistical analysis. Environment and Planning $A$ 23: $1,025-44$.

Fotheringham, A. S., P. A. Densham, and A. Curtis. 1995. The zone definition problem in locationallocation modeling. Geographical Analysis 27: 61-77.

Gehlke, C. E., and K. K. Biehl. 1934. Certain effects of grouping upon the size of the correlation coefficient in census tract material. Fournal of the American Statistical Association, Supplement 29: 169-70.

Golledge, R. G., and R. J. Stimson. 1987. Analytical Behavioral Geography. London: Croom Helm.

Golledge, R. G., T. R. Smith, J. W. Pellegrino, S. Doherty, and S. P. Marshall. 1985. A conceptual model and empirical analysis of children's acquisition of spatial knowledge. Fournal of Environmental Psychology 5: 125-52.

Gould, P. 1975. Acquiring spatial information. Economic Geography 51: 87-99.

1976. Cultivating the garden: A commentary and critique on some multidimensional speculations. In Spatial Choice and Spatial Behavior, ed. R. G. Golledge and G. Rushton, 83-91. Columbus: Ohio State University Press.

Harman, E. J., and J. F. Betak. 1976. Behavioral geography, multidimensional scaling and the mind. In Spatial Choice and Spatial Behavior, ed. R. G. Golledge and G. Rushton, 3-22. Columbus: Ohio State University Press.

Johnston, R. J., D. Gregory, and D. M. Smith, eds. 1991. Dictionary of Human Geography. Oxford: Blackwell.

Kitchin, R. M. 1994a. Cognitive maps: What are they and why study them? Fournal of Environmental Psychology 14: 1-19.

—. 1994b. CMAP (Cognitive Mapping Analysis Package). Public Domain Software, University of Wales Swansea.

Kitchin, R. M., and R. D. Jacobson. Forthcoming. Techniques to collect and analyze the cognitive map knowledge of people with visual impairments or blindness: Issues of validity. Fournal of Visual Impairment and Blindness.

Lloyd, R. 1989. Cognitive maps: Encoding and decoding information. Annals of the Association of American Geographers 79(1): 101-24.

Lloyd, R., and C. Heivly. 1987. Systematic distortions in urban cognitive maps. Annals of the Association of American Geographers 77: 191-207.

MacKay, D. B. and R. W. Olshavsky. 1975. Cognitive maps of retail locations: An investigation of some basic issues. Fournal of Consumer Research 2: 197-205. 
Magana, J. R., G. W. Evans, and A. K. Romney. 1981. Scaling techniques in the analysis of environmental cognition data. Professional Geographer 33: 294-301.

McNamara, T. P., J. K. Hardy, and S. C. Hirtle. 1989. Subjective hierarchies in spatial memory. Fournal of Experimental Psychology: Learning, Memory and Cognition 15: 271-87.

Neprash,J.A. 1934. Some problems in the correlation of spatially distributed variables. Fournal of the American Statistical Association, Supplement 29: 167-68.

Newcombe, N. 1985. Methods for the study of spatial cognition. In The Development of Spatial Cognition, ed. R. Cohen, 1-12. Hillsdale, NJ: Erlbaum.

Openshaw, S. 1983. The Modifiable Areal Unit Problem. CATMOG Series \#38. London: Institute of British Geographers.

Tobler, W. R. 1965. Computation of the correspondence of geographic patterns. Papers and Proceedings of the Regional Science Association 15: 131-39.
Turk, A. G. 1990. Towards an understanding of human-computer interaction aspects of geographic information systems. Cartography 19: 31-60.

Waterman, S., and D. Gordon. 1984. A quantitativecomparative approach to analysis of distortion in mental maps. Professional Geographer 36: 326-37.

ROBERT M. KITCHIN is a Lecturer in the School of Geosciences, Queen's Univerity of Belfast, Northern Ireland, United Kingdom BT7 1NN. His research interests include human spatial behavior, geography and disadvantage, and the geographical applications and implications of computing.

A. STEWART FOTHERINGHAM is Professor of Quantitative Geography in the Department of Geography, University of Newcastle, Newcastle, United Kingdom NE1 7RU. His research interests include spatial choice modeling, migration, and spatial statistics.

\title{
Evaluation of a Model for Predicting Simultaneous Contrast on Color Maps*
}

\author{
Cynthia A. Brewer \\ Pennsylvania State University \\ Perceptions of colors of individual map symbols are affected by simultaneous contrast, or induction, from surrounding \\ colors. In an experiment with human subjects, reaction times were significantly slower, with significantly more errors, \\ for test maps on which an induction model predicted that colors would be misinterpreted. Reaction times and error \\ rates improved significantly with map color adjustments that the model predicted would remove induced confusions. \\ These results represent progress toward establishing objective guidance for the design of effective color schemes in \\ computer-assisted mapping environments common in cartography, geographic information systems, and scientific \\ visualization. Key Words: cartography, color, simultaneous contrast, induction.
}

\section{Introduction}

M

ost visual displays produced using software for mapping, geographic information systems, and scientific visualization are rendered in color on CRTs. Researchers endeavor to choose color schemes for these displays that are readily understood. This effort is partly guided by choosing colors with perceptual ordering that emphasizes the logical ordering of data categories

* This research was supported by a Research, Scholarship and Creative Activity grant and an Affirmative Action Faculty Development Program grant from the College of Arts and Letters, San Diego State University. Cheryl Rogers assisted with the experiment reported in this paper. Eva Frank assisted with an earlier dissertation experiment that functioned as a pilot test. Dr. Judy Olson's role as advisor to the dissertation is acknowledged with appreciation. The dissertation research was supported by National Science Foundation Grant Number SES-8915175. Recommendations on analysis methods by an anonymous reviewer improved the paper. 